\title{
The circular RNA landscape in specific peripheral blood mononuclear cells of critically ill patients with sepsis
}

Hina N. Khan ${ }^{1,2}$, Xanthe Brands' ${ }^{1}$, Simona Aufiero², Arie J. Hoogendijk¹, Augustijn M. Klarenbeek', Tjitske S. R. van Engelen', Bastiaan W. Haak', Lonneke A. van Vught ${ }^{1}$, Janneke Horn ${ }^{3}$, Marcus J. Schultz ${ }^{3,4}$, Aeilko H. Zwinderman ${ }^{2}$, Tom van der Poll ${ }^{1,5}$ and Brendon P. Scicluna ${ }^{1,2^{*}}$ (D)

\begin{abstract}
Background: Dysregulation of the host immune response is a pathognomonic feature of sepsis. Abnormal physiological conditions are understood to shift efficient linear splicing of protein-coding RNA towards noncanonical splicing, characterized by the accumulation of non-coding circularized (circ)RNA. CircRNAs remain unexplored in specific peripheral blood mononuclear cells (PBMCs) during sepsis. We here sought to identify and characterize circRNA expression in specific PBMCs of patients with sepsis due to community-acquired pneumonia (CAP) relative to healthy subjects.

Methods: The study comprised a discovery cohort of six critically ill patients diagnosed with sepsis due to community-acquired pneumonia and four (age, gender matched) healthy subjects. PBMCs were isolated, and fluorescence-activated cell sorting was used to purify CD14+ monocytes, CD4+, CD8+ T cells, and CD19+ B cells for RNA sequencing. CD14+ monocytes from independent six healthy volunteers were purified, and total RNA was treated with or without RNase R.

Results: RNA sequencing of sorted CD14+ monocytes, CD4+, CD8+ T cells, and CD19+ B cells from CAP patients and healthy subjects identified various circRNAs with predominantly cell-specific expression patterns. CircRNAs were expressed to a larger extent in monocytes than in CD4+, CD8+ T cells, or B cells. Cells from CAP patients produced significantly higher levels of circRNA as compared to healthy subjects. Considering adjusted $p$ values, circVCAN (chr5:83519349-83522309) and circCHD2 (chr15:93000512-93014909) levels in monocytes were significantly altered in sepsis. Functional inference per cell-type uncovered pathways mainly attuned to cell proliferation and cytokine production. In addition, our data does not support a role for these circRNAs in microRNA sequestration. Quantitative PCR analysis in purified monocytes from an independent group of healthy volunteers confirmed the existence of circVCAN and circCHD2.

(Continued on next page)
\end{abstract}

\footnotetext{
* Correspondence: b.scicluna@amsterdamumc.nl

${ }^{1}$ Center for Experimental Molecular Medicine, Amsterdam University Medical Centers, location Academic Medical Center, University of Amsterdam, Room G2-105, Meibergdreef 9, 1105 AZ Amsterdam, the Netherlands

${ }^{2}$ Department of Clinical Epidemiology, Biostatistics and Bioinformatics,

Amsterdam University Medical Centers, location Academic Medical Center, University of Amsterdam, Amsterdam, the Netherlands

Full list of author information is available at the end of the article
}

(C) The Author(s). 2020 Open Access This article is licensed under a Creative Commons Attribution 4.0 International License, which permits use, sharing, adaptation, distribution and reproduction in any medium or format, as long as you give appropriate credit to the original author(s) and the source, provide a link to the Creative Commons licence, and indicate if changes were made. The images or other third party material in this article are included in the article's Creative Commons licence, unless indicated otherwise in a credit line to the material. If material is not included in the article's Creative Commons licence and your intended use is not permitted by statutory regulation or exceeds the permitted use, you will need to obtain permission directly from the copyright holder. To view a copy of this licence, visit http://creativecommons.org/licenses/by/4.0/ The Creative Commons Public Domain Dedication waiver (http://creativecommons.org/publicdomain/zero/1.0/) applies to the data made available in this article, unless otherwise stated in a credit line to the data. 
(Continued from previous page)

Conclusions: We provide a benchmark map of circRNA expression dynamics in specific immune cell subsets of sepsis patients secondary to CAP. CircRNAs were more abundant in immune cells of sepsis patients relative to healthy subjects. Further studies evaluating circRNA expression in larger cohorts of sepsis patients are warranted.

Keywords: Circular RNA, Peripheral blood mononuclear cells, Community-acquired pneumonia, Sepsis

\section{Background}

Sepsis is a complex syndrome initiated by an imbalanced reaction to infection, with community-acquired pneumonia (CAP) as a major cause, leading to organ damage and high mortality rates [1, 2]. Despite advances in critical care medicine and antimicrobial therapy, no drug specifically targeting sepsis has been approved [3]. The immunopathology of sepsis is understood to encompass a plethora of host defense reactions that are primarily categorized as either abnormally excessive or suppressed [4]. Excessive inflammation (or hyperinflammation) exacerbates organ dysfunction whereas immune suppression induces immune cells to become perpetually unresponsive to pathogens, thereby increasing the risk of mortality due to, for example, uncontrolled secondary infections and failure to restore homeostasis [5]. Prospective observational cohorts to study the genomics of sepsis have shown that patients present signatures of both sustained inflammation and immune suppression [68]. Identifying regulatory features of the genomic response in sepsis is crucial for future drug discovery.

Advances in next-generation sequencing and bioinformatics have aided in the identification of a novel type of RNA class that is naturally resistant to degradation by exonucleases, termed circular (circ)RNA [9]. CircRNAs are single-stranded RNA molecules formed by noncanonical pre-messenger (m) RNA splicing, where downstream donor sites are "back-spliced" to upstream acceptor sites (e.g., the end of exon 4 covalently binds to the start of exon 4) [10]. In general, the abundance of circRNA is low when compared to the associated linear mRNA [11-13], suggesting that the formation of circRNA may be inefficient for cellular physiology [14]. While the functions of circRNA remain largely unknown, proposed roles include sequestration of microRNA, protein binding, and interference in transcript splicing [15]. The patterns of circRNA expression in peripheral blood mononuclear cells (PBMCs) of critically ill patients with sepsis remain unexplored. Here, we sought to characterize the circRNAome in specific PBMCs of sepsis patients secondary to CAP relative to healthy subjects. Our findings provide a benchmark in understanding the role of circRNA in the genomic response during sepsis secondary to CAP.

\section{Methods}

\section{Study population}

The study comprised a discovery cohort of six critically ill patients with sepsis due to community-acquired pneumonia (CAP) with positive blood cultures for Streptococcus pneumoniae (S. pneu) selected from within the framework of the Molecular diAgnosis and Risk stratification in Sepsis (MARS) project (ClinicalTrials.gov identifier NCT01905033) [16, 17]. Sepsis diagnosis was described in detail previously $[16,18]$. This cohort was enrolled between January 2011 and July 2012. The Medical Ethics committees approved an opt-out consent method (IRB no. 10-056C). Severity was assessed by Acute Physiology and Chronic Health Evaluation (APAC HE) IV [19] and total Sequential Organ Failure Assessment (SOFA) scores excluding the central nervous system component [20]. Shock was qualified by the use of vasopressors (norepinephrine, epinephrine, or dopamine) for hypotension in a norepinephrine-equivalent dose of more than $0.1 \mu \mathrm{g} / \mathrm{kg} / \mathrm{min}$ [2]. Four healthy subjects (age and gender matched) were also included in the discovery cohort. A total of six independent healthy volunteers ( $>18$ years old) were also included for in vitro validation assays. From all healthy subjects, written informed consent was obtained.

\section{Peripheral blood mononuclear cell isolation}

Heparinized whole blood $(10 \mathrm{ml})$ was collected from healthy subjects and sepsis patients. Blood from sepsis patients was collected within $24 \mathrm{~h}$ of ICU admission and diluted 1:1 with phosphate-buffered saline (PBS) (Fresenius Kabi). PBMCs were separated by centrifugation using Ficoll-Paque PLUS (GE Healthcare Life science, Little Chalfont, UK) followed by treatment with ice-cold erythrocyte lysis buffer (Qiagen, Venlo, The Netherlands) and washed twice with ice-cold PBS supplemented with $0.5 \%$ sterile endotoxin-free bovine serum albumin (BSA; Divbio Science Europe, AK8917-0100). PBMCs were stained with cluster of differentiation (CD)14-APC-Cy7, CD3-AlexaFluor700, CD4-PERCP-Cy5.5, CD15-FITC, CD20-PE-CF594 (BD Biosciences), and CD8-APC (eBioscience, San Diego, CA) for $30 \mathrm{~min}$ at $4{ }^{\circ} \mathrm{C}$. Within a lymphocyte gate based on forward and side scatter, CD4 $\mathrm{T}$ cells were defined as $\mathrm{CD} 3^{+} \mathrm{CD} 4^{+} \mathrm{CD} 8^{-}, \mathrm{CD} 8 \mathrm{~T}$ cells as $\mathrm{CD}^{+} \mathrm{CD}^{+} \mathrm{CD}^{-}$, and $\mathrm{B}$ cells as $\mathrm{CD}^{-} \mathrm{CD} 19^{+}$. In the 
monocyte gate, monocytes were identified as $\mathrm{CD} 14^{+} \mathrm{CD} 15^{-}$cells. Data acquisition was performed using a FACSCanto II flow cytometer (BD Biosciences). After sorting, cells were kept in RNAprotect Cell reagent (Qiagen, Venlo, The Netherlands \#76526). Monocytes were also captured from six healthy subjects enrolled for in vitro validation assays. Healthy PBMCs were purified from heparinized whole blood using Ficoll-Paque as described above, and monocytes were purified with magnetic beads coated with anti-CD14 antibody respectively (Miltenyi Biotech). Monocyte purity was verified by flow cytometry (> 95\% CD $14^{+} \mathrm{CD} 15^{-}$cells). Flow cytometry data were analyzed with Flowjo X.07 (Tree Star, Ashland, OR).

\section{RNA sequencing}

Total RNA was isolated from each cell type by means of the miRNeasy RNA isolation kit (Qiagen, Venlo, The Netherlands) according to the manufacturer's instructions. RNA quality was assessed by bioanalysis (Agilent), with all samples having RNA integrity numbers (RINs) $>7$. Total RNA concentrations were determined by Qubit $^{\oplus} 2.0$ Fluorometer (Life Technologies, Carlsbad, CA, USA). Sequencing libraries were prepared by means of the Ovation $^{\curvearrowleft}$ RNA-Seq System V2 (NuGEN) kit as per the manufacturer's instructions. Libraries were sequenced using the Illumina HiSeq2500 (Illumina) to generate $2 \times$ 126 bp paired-end (PE) reads. Sequence read quality was assessed by means of the FastQC methods (version 0.11.5; Babraham Institute, Babraham, Cambridgeshire, UK). Trimmomatic version 0.32 [21] was used to trim the Illumina adapters containing poor-quality bases and ambiguous nucleotide containing sequences. Low-quality leading (3 nucleotides) and trailing (3 nucleotides) bases were removed from each read, and the quality of the body of the reads was assessed with a sliding window trimming using a window of 4 and a phred score threshold of 15 nucleotides. After pre-processing, the remaining high-quality reads were used to align against the Genome Reference Consortium Human Genome Build 38 patch release 7 (GRCh38.p7) [22]. Mapping was performed by Tophat2 version 2.1.1 [23] with parameters as default. Count data were generated by means of the featureCounts method [24] and analyzed using the DESeq2 method [25] in the R statistical computing environment ( $\mathrm{R}$ Core Team 2014. R: A language and environment for statistical computing. $\mathrm{R}$ Foundation for Statistical Computing, Vienna, Austria). Significance was defined by Benjamini-Hochberg $(\mathrm{BH})$ adjusted $p$ value $\leq 0.05$ and fold change $\geq 1.5$ or $\leq-1.5$. The bioinformatics workflow is represented in Additional file 1: Fig. S1.

\section{Circular RNA bioinformatics}

Sequence reads were analyzed by Mapsplice2 [26] with the following parameters: --fusion-non-canonical, --min- fusion-distance 200, and --min-map-len 25. The short read aligner Bowtie [27] was used to align the reads to the reference genome (GRCh38.p7). A circRNA was called if it was supported by at least four back-spliced reads in at least two different samples. To perform reverse complementary sequence (RCS) analysis, we aligned the downstream and reversed complement of the upstream intron using the pairwiseAlignment function implemented in the Biostrings $R$ package [28]. The identified significant [29] RCSs were then blasted using the RepeatMasker program [30] to screen sequences for interspersed repeats and low complexity DNA sequences. The flanking intron sequences of all circRNAs were obtained from the GENCODEv25.p7 human genome reference. The RNA-hybrid tool [31] was used to predict putative micro (mi) RNA target sites in circRNA. To determine the relative expression of circRNA with respect to the host linear RNA, we used the backsplice-to-linear ratio as described previously [32], modified by taking the average of read counts for all samples $(S 1 \ldots S n)$.

$$
\begin{aligned}
& \text { Back - splice }- \text { to }- \text { linear ratio }_{(\text {Gene })} \\
& =\frac{\left.\operatorname{Average}_{(S 1 \ldots S n)}\right)}{\operatorname{Max}\left(c_{(\text {Average }}\left(l 1_{(S 1 \ldots S n)}\right), \operatorname{Average}\left(l 2_{(S 1 \ldots S n)}\right)\right)}
\end{aligned}
$$

where $c$ is total read count of circRNA back-splice junction and $l 1$ and $l 2$ represent the total linear read count that spans left and right linear-spliced junctions of the same exon(s), respectively.

For miRNA enrichment analysis, a cumulative distribution function (CDF) was computed using the ecdf function of the R Bioconductor package, Stats. The University of California Santa Cruz (UCSC) table browser [33] and data integrator tool along with custom tracks were used to obtain the coordinates and exon sequences. miRbase (release 22) was used to get the human mature miRNA sequences. Pathway analysis of the circRNA host genes was done by gene ontology (GO) enrichment analysis [34] and Ingenuity pathway analysis (IPA; Qiagen bioinformatics). Human species and IPA gene knowledgebase were selected. All other settings were default. $\mathrm{BH}$ multiple-test adjusted $p$ values $<0.05$ defined significance.

\section{Validation assay}

CD14+ monocytes purified from six healthy volunteers were seeded at $0.5 \times 10^{6}$ cells per well with Roswell Park Memorial Institute (RPMI) medium supplemented with $10 \%$ sterile fetal calf serum (FCS; HyClone, \#SV30160.03), $200 \mathrm{mM}$ glutamax (Thermo Fisher, \#35050-038), $100 \mu \mathrm{M}$ pyruvate (Thermo Fisher, \#11360-039), and $50 \mu \mathrm{g} / \mathrm{ml}$ gentamycin (Lonza, \#17-5192) in a cell-repellent surface 48well plate (Greiner Bio-one, \#677970). Total RNA was 
isolated from purified monocytes using the RNeasy Mini Kit (Qiagen, \#74104) according to the manufacturer's instructions. RNA quality and concentration were assessed using Nanodrop (Thermo Fisher). To generate RNase R digested RNA [35], $150 \mathrm{ng}$ total RNA was incubated in $1 \mathrm{x}$ RNase $\mathrm{R}$ buffer in a 20- $\mu \mathrm{l}$ reaction with or without 5 units of RNAse R (Epicentre) at $37^{\circ} \mathrm{C}$ for $10 \mathrm{~min}$ followed by heat inactivation at $95^{\circ} \mathrm{C}$ for $3 \mathrm{~min}$. DNA was depleted using DNase I (Invitrogen, \#79254). Complementary DNA (cDNA) was synthesized with random primers using the SuperScript III reverse transcriptase (RT) kit (Invitrogen; \#11752250) as per the manufacturer's instructions. Divergent primers were designed for the versican (VCAN; chromosome (chr)5: 83519349-83522309) and chromodomain helicase DNA binding protein 2 (CHD2; chr15: 93000512-93014909) loci (Additional file 2: Fig. S2). The sequences of $V C A N$ circRNA primers were $5^{\prime}$-GCCC CCAGCAAGCACAAAATTT-3' (forward) and 5' -TGCA GTTTCTGCGAGGATACTC-3' (reverse). The sequences of the CHD2 circRNA primers were $5^{\prime}$-TCACCCCAAC AAGAGACACTTC-3' (forward) and 5'-TCTTTCAGCC TGGGCACTTTGT-3' (reverse). The hypoxanthine phosphoribosyltransferase (HPRT) gene was used as linear messenger (m) RNA control. The sequences of the HPRT primers were 5'-GGATTTGAAATTCCAGACAAGTTT3' (forward) and 5'-GCGATGTCAATAGGACTCCAG3' (reverse). Quantitative reverse-transcriptase polymerase chain reaction (qRT-PCR) was performed by using the SensiFAST SYBR No-ROX Mix (Bioline, \#CSA-01190) and a LightCycler480 system II (Roche) using the following program: $95^{\circ} \mathrm{C}$ pre-incubation for $6 \mathrm{~min}$ followed by 40 cycles of $95^{\circ} \mathrm{C}(10 \mathrm{~s}), 62^{\circ} \mathrm{C}(20 \mathrm{~s})$, and $72{ }^{\circ} \mathrm{C}(20 \mathrm{~s})$. RTPCR products were separated by agarose gel $(2 \%$, Roche \#11388991001) electrophoresis, and bands were visualized by Syngene Gbox scanner. Data were quantified and analyzed by means of the LinRegPCR method [36]. CircRNA expression indices of treated samples were normalized to corresponding linear HPRT1 expression in untreated samples.

\section{Monocyte stimulation}

In order to evaluate the inducibility of circRNA, monocytes from healthy participants were stimulated with lipopolysaccharide (LPS; EB Ultrapure Invivogen \#tlrl3pelps), heat-killed Streptococcus (S.) pneumoniae (Sp; ATCC6303), and Klebsiella (K.) pneumoniae (Kp; ATCC43816) at $37^{\circ} \mathrm{C}$ with $5 \% \mathrm{CO}_{2}$ and $95 \%$ humidity for 2 and $24 \mathrm{~h}$. Heat-killed bacteria $\left(70^{\circ} \mathrm{C}\right.$ for $\left.30 \mathrm{~min}\right)$ were used at a bacteria-to-cell ratio of 10:1. Supernatants were collected for enzyme-linked immunosorbent assays (ELISAs). Tumor necrosis factor alpha (TNF) and interleukin (IL)-6 levels were measured using commercially available ELISA kits (TNF, R\&D systems \#MAB610; IL6, Thermo Fisher \#88710677). Bradford protein assay
(BIO-RAD, Hercules, CA) was used for total protein measurements. Cells were resuspended in RNAprotect Cell reagent (Qiagen, Venlo, The Netherlands \#76526) and stored at $-80^{\circ} \mathrm{C}$ prior to qRT-PCR analysis.

\section{Statistics}

The length of exons involved in circRNAs, introns flanking the expressed circRNAs, and the control set were compared using an unpaired Student $t$ test. The paired Student $t$ test was used to compare the circRNA expression with its linear counterpart. Quantitative RT-PCR data was analyzed by Wilcoxon rank-sum test. Unless otherwise stated, $p<0.05$ demarcated significance. Heat maps were generated using the pheatmap $\mathrm{R}$ method, and principal component analysis (PCA) was done using the FactoMinerR $\mathrm{R}$ package. Venn diagrams were made using the VennDiagram R package.

\section{Results \\ Circular RNA detection and characterization in CAP and health}

RNA-sequencing data of monocytes, $\mathrm{CD} 4^{+}, \mathrm{CD} 8^{+} \mathrm{T}$ cells, and $\mathrm{B}$ cells isolated from healthy subjects (median age [Q1-Q3], 54 [50-60]; males [\%], 2 [50]) and CAP patients were used to map circRNA (Fig. 1a). The CAP patients consisted of elderly adults (median age [Q1-Q3], 62 [5068]; males [\%], 1 [17]) with median APACHE IV score of 52 (Q1-Q3, 47-74), median SOFA score of 6 (Q1-Q3, 5$8), 50 \%$ having shock, and $67 \%$ on mechanical ventilation. In total, 734, 753, 636, and 430 circRNAs were identified in monocytes, CD4+, CD8+ T cells, and B cells, respectively (Additional file 3: Table S1). PCA of circRNA expression revealed clearly distinct clusters per cell type with explainable variance of $12.8 \%$ (PC1) and $8.7 \%$ (PC2) (Fig. 1b). On average, circRNAs were expressed to a significantly lower extent than the corresponding host gene expression (Fig. 1c). Monocytes produced more circRNA than CD4+, CD8+ T cells, or B cells, with back-splice-tolinear ratios (a measure of circRNA expression relative to linear mRNA counterpart) equating to $0.13(\mathrm{Q} 1-\mathrm{Q} 3$, 0.05-0.39), 0.06 (Q1-Q3, 0.05-0.26), 0.07 (Q1-Q3, 0.06$0.29)$, and $0.05(\mathrm{Q} 1-\mathrm{Q} 3,0.02-0.06)$, respectively. The gene encoding nucleolin $(N C L)$ produced the most circRNA species (total of 13), polyhomeotic homolog 3 (PHC3) produced 12 circRNAs, and RNA binding motif protein 25 (RBM25) produced 11 circRNAs (Fig. 1d). The longest predicted circRNA encompassed 44 exons of the centrosomal protein 192 (CEP192) gene (chr18: 1299942113117643) (Fig. 1e). Of note, while the average length of circRNA exons was not longer than the genome-wide average (Fig. 1f), flanking introns were significantly longer (Fig. 1g). Furthermore, the flanking introns of the predicted circRNAs were significantly enriched for RCSs, containing interspersed Arthrobacter luteus (Alu) 


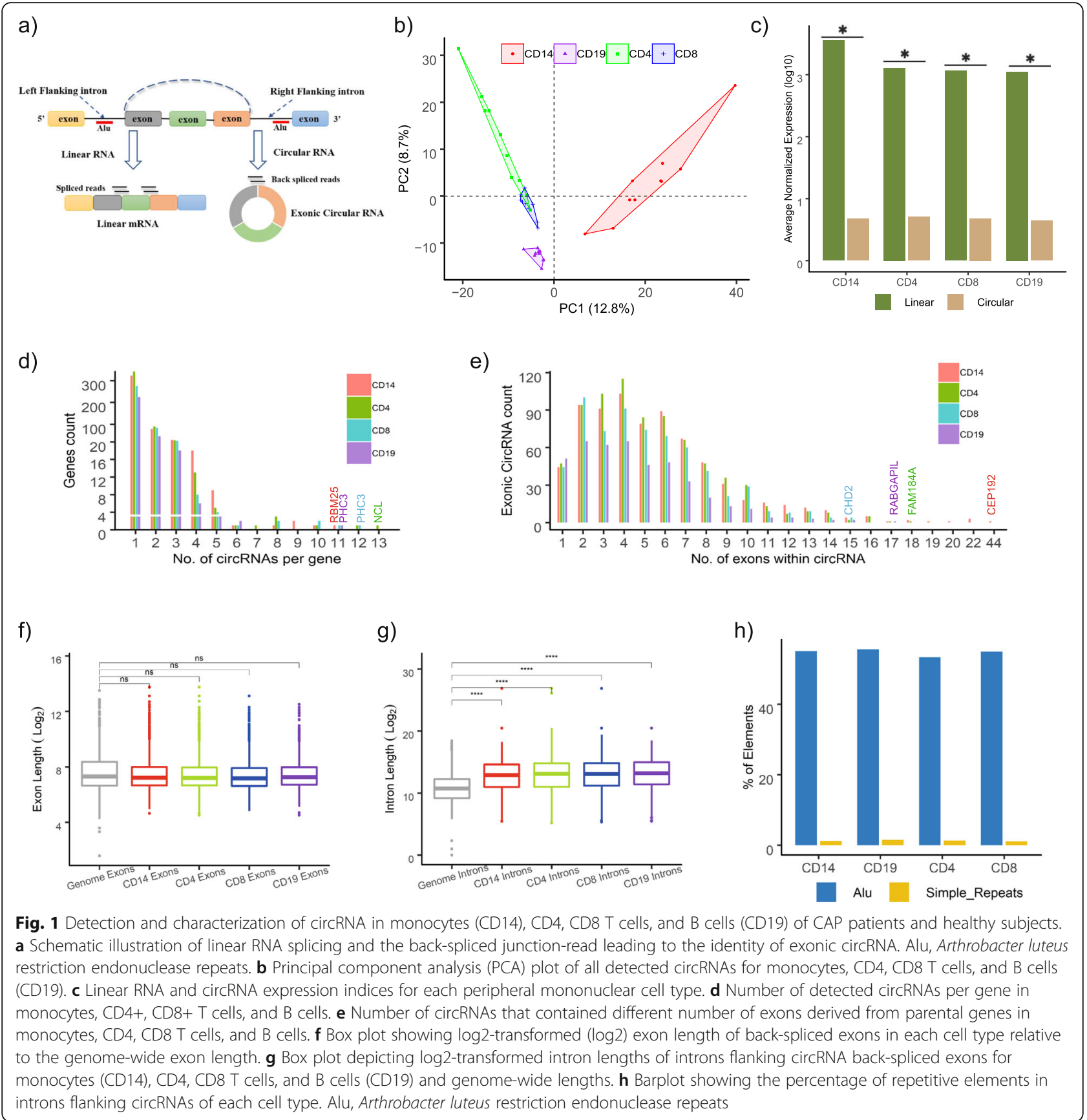

restriction endonuclease repeats (55\%) and simple repeats (1.3\%) (Fig. 1h). These findings are consistent with genomic features that convey RNA circularization [13-15]. Altogether, circRNAs were produced by each immune cell type in both healthy individuals and CAP patients. Monocytes were predicted to produce substantially more circRNAs than T or B cells.

Circular RNA expression patterns in CAP relative to health Here, we sought to identify significantly altered circRNA expression in monocytes, $\mathrm{T}$ cells, and $\mathrm{B}$ cells of CAP patients relative to healthy samples. In general, cells of CAP patients produced more circRNA than healthy participants (Fig. 2a). Considering multiple-comparison adjusted $p$ values, circRNAs from the versican gene (VCAN; chr5: 83519349-83522309) and chromodomain helicase DNA binding protein 2 gene (CHD2; chr15: 93000512-93014909) were significantly more abundant in monocytes of CAP patients (Fig. 2b, c). A list of detected and altered circRNA can be found in Additional file 3: Table S1. CircRNA expression differences between study groups were largely unique to cell type, and none 
a)

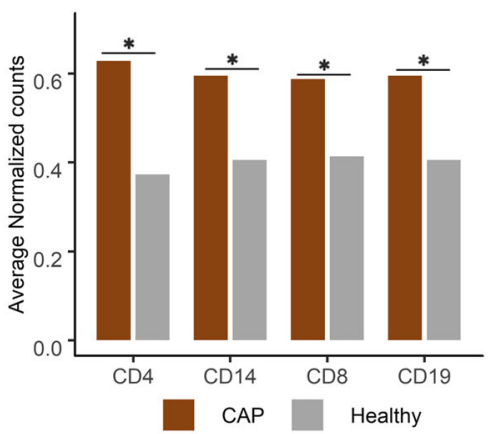

b)

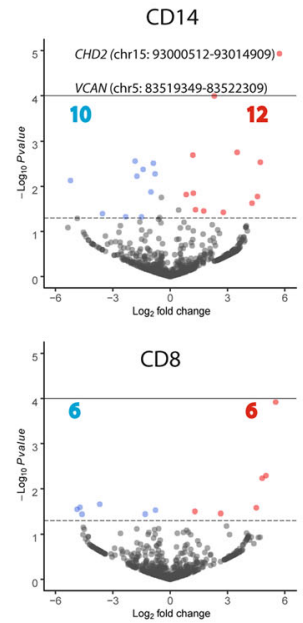

CD4

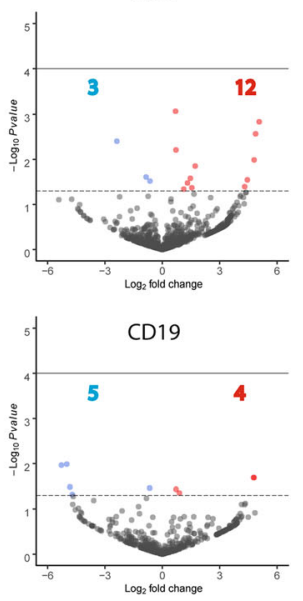

c)
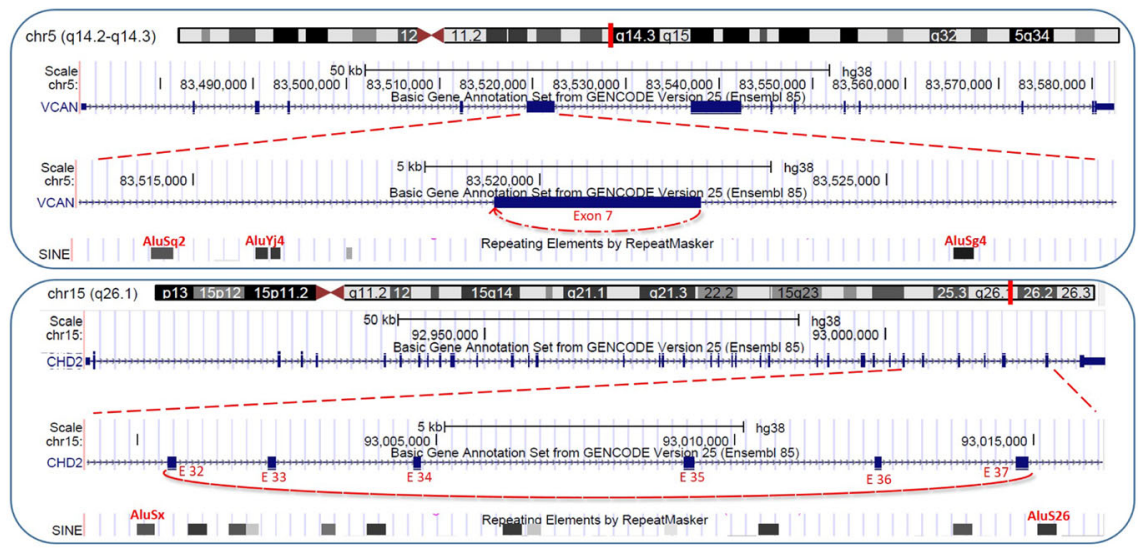

d)

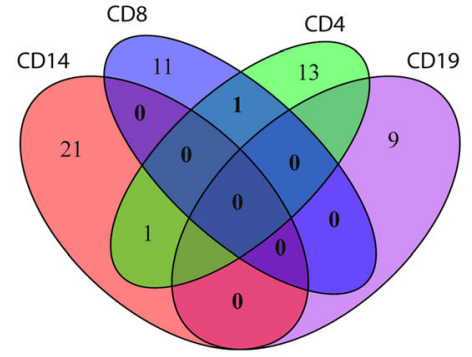

e)

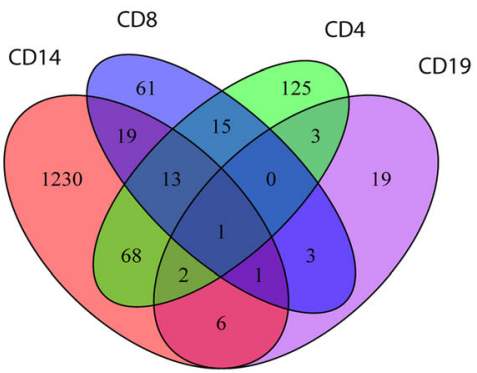

Fig. 2 Circular RNA expression in patients with sepsis due to CAP relative to healthy subjects. a Barplot illustrating the average circRNA expression in monocytes (CD14), CD4, CD8 T cells, and B cells (CD19) of CAP patients and healthy volunteers. $\mathbf{b}$ Volcano plot representing the differentially expressed circRNA of each peripheral mononuclear cell type in CAP as compared to the health. The horizontal dotted line represents the nominal $p$ value $\leq 0.05$ threshold. A horizontal solid line represents the multiple-comparison adjusted $p$ value $\leq 0.05$ threshold. Red dots, upregulated circRNA (nominal $p<$ 0.05); blue dots, downregulated circRNA (nominal $p<0.05$ ); gray dots, not altered circRNA (nominal $p>0.05$ ). c Representative UCSC genome browser of the exons corresponding to the two significantly altered (adjusted $p$ value $<0.05$ ) circRNAs in monocytes of CAP patients relative to healthy patients. The presence of Alu repeats in the flanking introns is noted. E, exon; Alu, Arthrobacter luteus restriction endonuclease repeats. $\mathbf{d}$ Venn diagram of differentially abundant circRNAs (nominal $p<0.05$ ) per cell type. e Venn diagram of differentially expressed (adjusted $p<0.05$ ) linear mRNA species in monocytes, CD4, CD8 T cells, and B cells (CD19) of CAP patients relative to healthy subjects

showed constitutive alterations in all cells (Fig. 2d). This observation was also evident for linear RNA species with predominantly cell-type-specific expression patterns in CAP patients compared to healthy subjects (Fig. 2e and Additional file 4: Fig. S3). Of the 1340, 227, 113, and 35 significantly altered genes (adjusted $p<0.05$ ) in monocytes, CD4+, CD8+ $\mathrm{T}$ cells, and $\mathrm{B}$ cells, respectively (Additional file 4: Fig. S3), 1230, 125, 61, and 19 genes were specifically altered in respective cell types (Fig. 2e). Notably, VCAN expression in monocytes was 
significantly higher in CAP relative to health (adjusted $p=0.0013)$. No differences were detected for CHD2 gene expression. Our findings showed largely cell-typespecific circRNA expression patterns and specific circRNA were significantly altered in monocytes of CAP patients relative to healthy subjects.

\section{Functional inference of circular RNA}

In order to infer on putative functions and potential biological roles of the altered circRNA identified in each cell type, we firstly evaluated cellular biological pathway enrichment of the "host" genes producing the circRNAs. Genes producing circRNAs that were differentially abundant in monocytes, $\mathrm{CD} 4+$, and CD8+ T cells of CAP patients (relative to healthy subjects) (Fig. $3 a-c$ ) were associated to distinct canonical signaling pathways (Fig. 3d). In particular, monocyte circRNA was primarily associated with Notch signaling and ERK signaling pathways. Numerous pathways were detected for low expression circRNA in CD4+ T cells, including FLT3 signaling in hematopoietic progenitor cells (Fig. 3d), as well as RAN signaling and UVC-induced MAPK signaling for CD8+ T cells. High expression circRNA was mainly associated with IL-15 production and DNA double-strand break repair by homologous recombination pathways (Fig. 3d). Secondly, motivated by reported functions of circRNA as miRNA "sponges" [13], we explored circRNA sequences for potential miRNA binding regions. The frequency of miRNA binding sites in circRNA exons was not different to all other exons in the genome that did not produce circRNA (Fig. 3e). Therefore, the lack of miRNA binding site enrichment in the herein predicted circRNA does not support their role as miRNA "sponges".

\section{Experimental validation}

Thus far, we have identified putative circRNA species, their expression patterns, and potential functional pathways in different peripheral blood mononuclear cell types from CAP patients and healthy volunteers. Here, we sought to provide robustness to our bioinformatics approach by experimental validation of circRNA existence in an independent population of healthy subjects. We focused our attention on the two significantly altered circRNAs in monocytes, that is, VCAN (chr5: 83519349-83522309; circVCAN) and CHD2 (chr15: 93000512-93014909; circCHD2). Prior to quantitative RT-PCR analysis, we treated total RNA from monocytes with or without the RNA digestion enzyme, RNase R. As expected, treatment with RNase $\mathrm{R}$ resulted in a reduction in HPRT1 transcripts (non-circular endogenous control) (Fig. 4a). In contrast, no reduction was observed for circVCAN and circCHD2 (Fig. 4a), which indicated resistance to RNase digestion. CircVCAN was more abundant than circCHD2 (Fig. 4b). These results provide robustness to the existence of circVCAN and circCHD2 in human monocytes.

Next, we evaluated the inducibility of circVCAN and circCHD2 by stimulating monocytes with LPS, as well as clinically relevant $K$. pneumoniae and S. pneumoniae. As expected, TNF and IL- 6 protein levels in the supernatants were significantly elevated after 2- and 24-h stimulation (Fig. 4c). In contrast, circVCAN and circCHD2 were not induced (Fig. 4d). Altogether, our findings provide benchmark evidence of the existence of circVCAN and circCHD2 in healthy human monocytes, which were not inducible by stimulation with LPS nor clinically relevant bacteria.

\section{Discussion}

Transcriptional activity of genes is not exclusive to production of linear mRNA molecules for translation to proteins. A variety of mature RNA species can be produced per gene, including thousands of circRNA. In the current study, we demonstrated the presence and quantified the abundance of circRNAs in specific PBMCs of sepsis patients secondary to CAP relative to healthy subjects. In general, circRNA production was significantly higher in CAP patients relative to healthy patients, and expression patterns were largely specific to either monocytes, $\mathrm{CD} 4^{+}, \mathrm{CD}^{+} \mathrm{T}$ cells, or B cells. Functional inference showed potential roles of circRNA in distinct pathways per cell type, including cytokine production and cell differentiation. Moreover, our data does not support a reported role of circRNA in sequestration of miRNA. High-confidence circRNA was detected in monocytes of an independent cohort of healthy volunteers by exoribonuclease RNase $\mathrm{R}$ digestion and quantitative RT-PCR. Altogether, our study provides a benchmark in the identification, characterization, and potential functional implications of the circRNAome in important immune cell types of sepsis patients secondary to CAP.

Animal genomes produce thousands of circRNA molecules in complex tissue, cell type, and developmental stage-specific ways [37]. CircRNA represents a novel class of RNA molecules produced by transcriptional "back-splicing" and composed of only exons, exon-introns, or only introns. Contrary to the past notion that circRNA represented by-products of splicing errors, reported functions include transcriptional regulation of protein-coding RNA. For example, circRNAs CDR1as (ciRS-7) and circ-SRY were shown to sequester miRNAs, thereby altering transcriptional outputs [37, 38]. Furthermore, recent studies suggest circRNA may not only function as non-coding transcriptional regulators but may also harbor potential for translation to amino acids, and ultimately proteins [39]. For instance, overexpression of circ-FBXW7 that 
a)

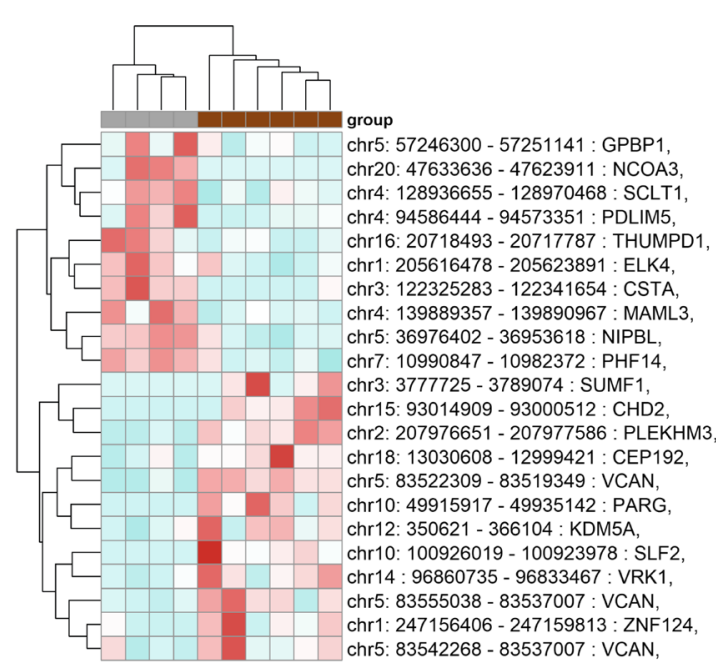

c)

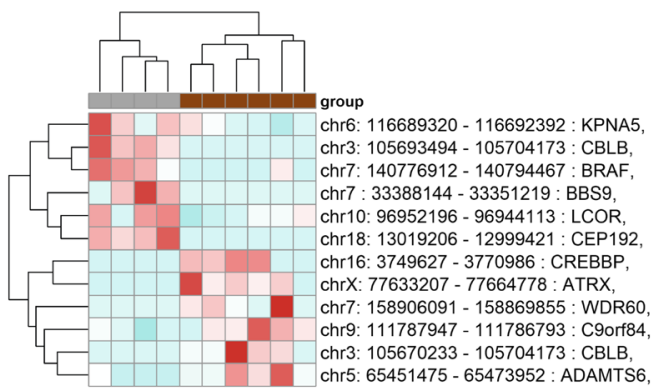

e)

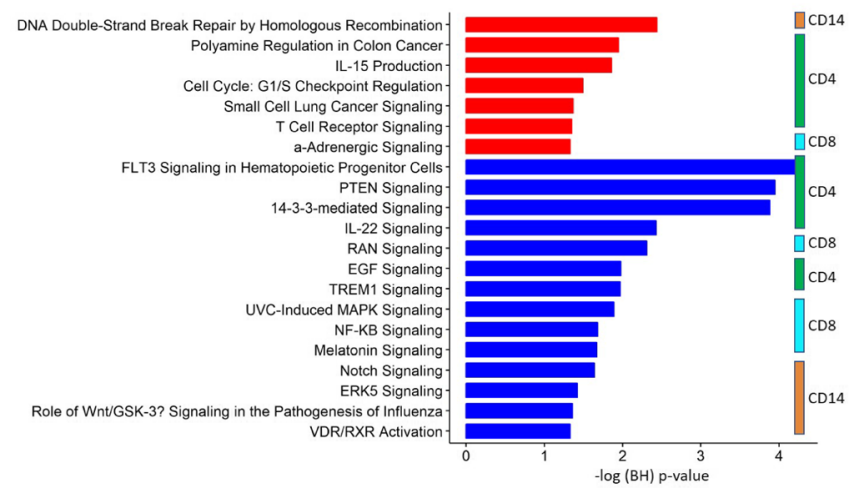

b)

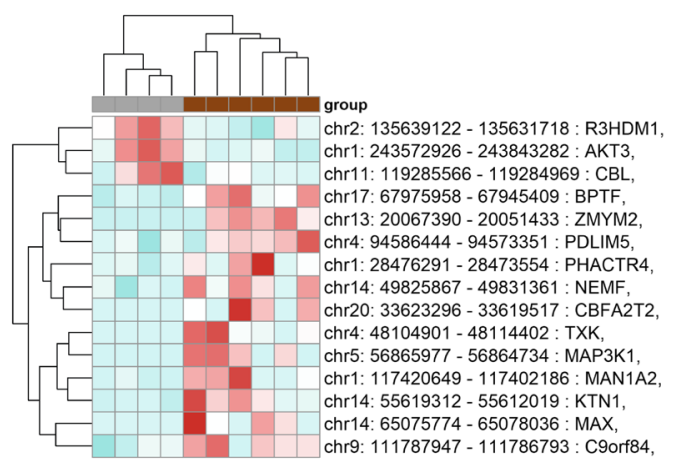

d)
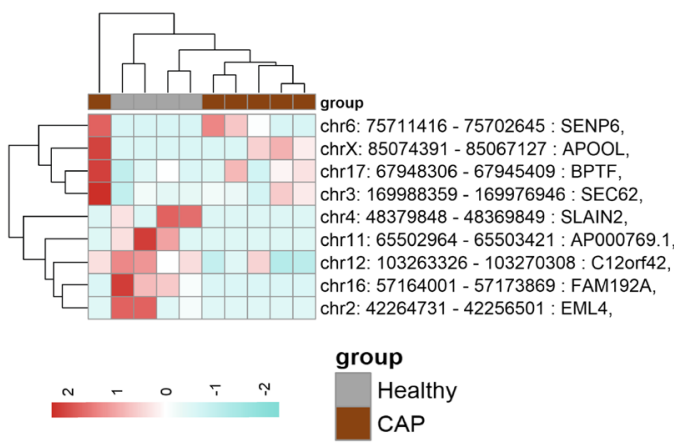

f)

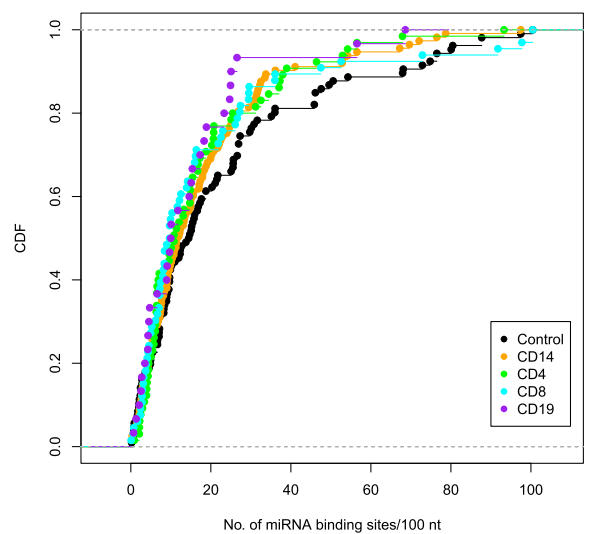

Fig. 3 Functional inference of circRNAs identified in monocytes (CD14), CD4, CD8 T cells, and B cells (CD19) of CAP patients and healthy subjects. a- $\mathbf{d}$ Heatmaps showing expression patterns of differentially altered circRNAs (nominal $p$ value $<0.05)$ in monocytes $(\mathbf{a}), C D 4+T$ cells $(\mathbf{b}), C D 8+T$ cells (c), and B cells (d) of CAP patients relative to healthy subjects. e Pathway analysis of the host genes producing the circRNAs for monocytes and CD4+, CD8+ T cells. The vertical axis represents the pathways, and the horizontal axis represents the -log 10-transformed Benjamini-Hochberg (BH) adjusted $p$ values. Red bars represent pathways for host genes of upregulated circRNA, whereas blue bars denote pathways for host genes of downregulated circRNA in CAP relative to health. Vertical bars depict the cell type, that is, monocytes (orange), CD4+ (green), and CD8+ (cyan). f Cumulative distribution function (CDF) showing the abundance of predicted micro (mi) RNA binding in the exons of differentially abundant circRNAs of monocytes (orange), CD4+ (green), CD8+ (cyan) T cells, and B cells (purple) and human host gene exons that do not circularize (controls, black) 
a)

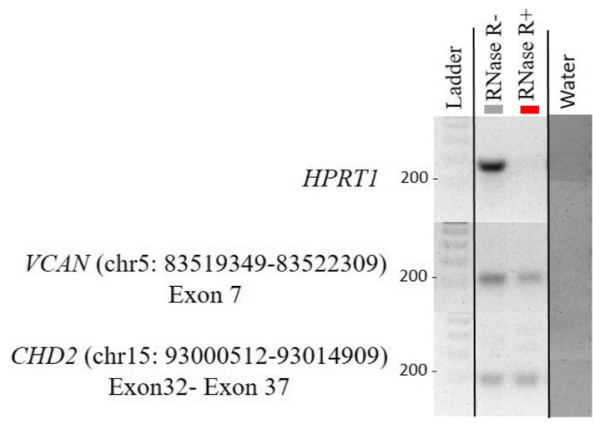

c)

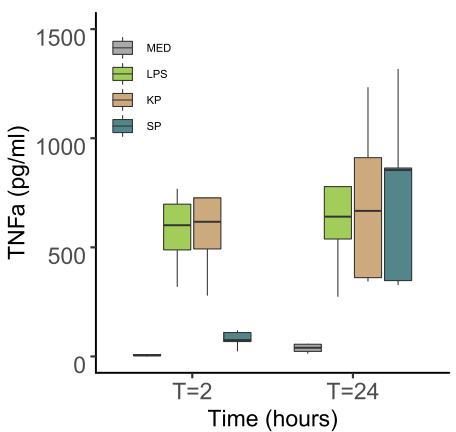

d)

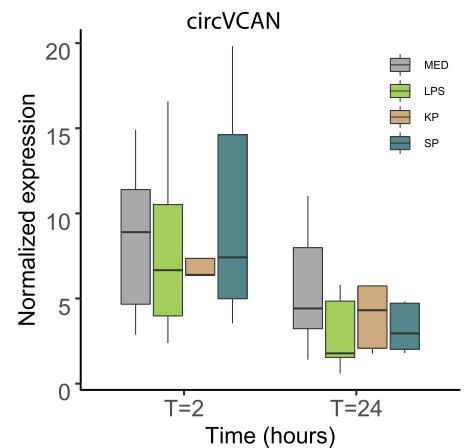

b)
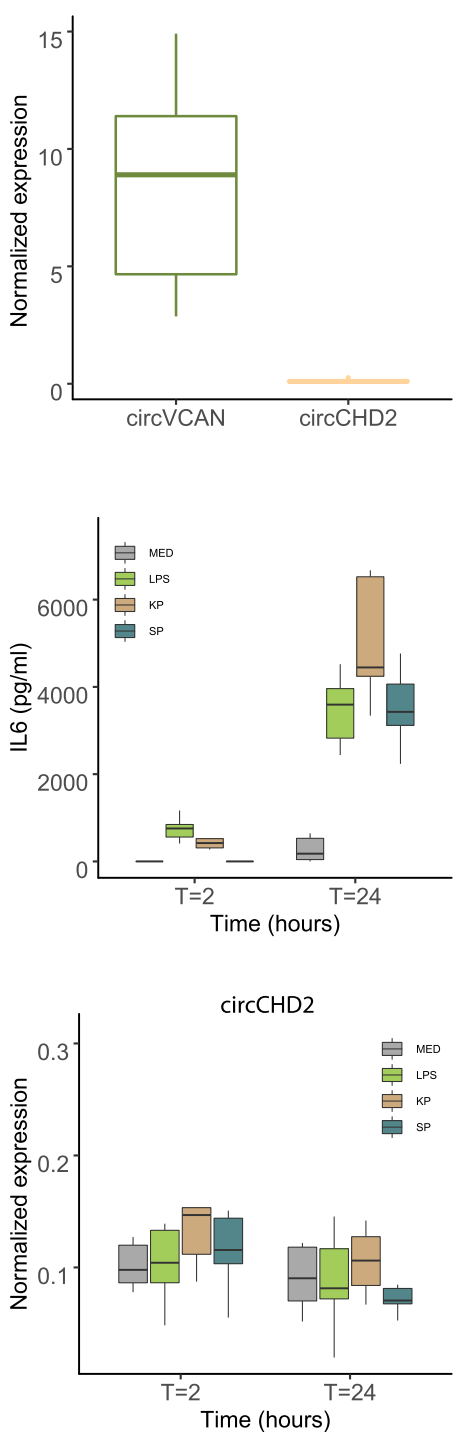

Fig. 4 Experimental validation of detected circRNAs in healthy CD14+ monocytes. a Agarose gel scan illustrating amplicons of linear mRNA (HPRT1) and two circRNAs VCAN (chr5: 83519349-83522309) and CHD2 (chr15: 93000512-93014909) treated with (+) or without (-) RNase R. Ladder, Thermo Scientific GeneRuler DNA Ladder mix \#SM0333. b Quantitative RT-PCR normalized expression patterns of VCAN (chr5: 8351934983522309) and CHD2 (chr15: 93000512-93014909) circRNAs in healthy monocytes. c Box plots depicting the production of TNF and IL-6 by monocytes stimulated with lipopolysaccharide (LPS), heat-killed Streptococcus pneumoniae (Sp), and Klebsiella pneumoniae (Kp) for 2 and $24 \mathrm{~h}$. ${ }^{*} p<0.05$. d Box plots illustrating the normalized expression patterns of circRNAs, circVCAN (chr5: 83519349-83522309) and circCHD2 (chr15: 93000512-93014909), in stimulated monocytes

encodes a novel $21 \mathrm{kDa}$ protein composed of 185 amino acids, named F-Box and WD Repeat Domain Containing 7 (FBXW7), repressed cellular proliferation and glioma tumorigenesis [40]. It is becoming evident that noncanonical transcriptional "back-splicing" can compete with canonical linear splicing that produces mRNA for translation to protein products [41]. In particular, conditions of cellular stress, for example, in drosophila inhibition of splicing machinery, were shown to shift the steady-state output of pre-mRNA splicing towards circularization [42]. Critical illness due to sepsis is associated with severe cellular stress, which oftentimes precipitates to a condition of "immunoparalysis," thereby making immune cells nonresponsive to secondary challenges $[4,7]$. The extent of circRNA effects on immune cell responsiveness and tolerance to bacterial ligands (e.g., endotoxin) certainly warrant exploration.

Assigning biological functions to circRNA is an emerging field of functional genomics. The use of bioinformatics tools and molecular biology can elucidate their function in cellular physiology and define their role in disease conditions. The circRNA-miRNA "sponge" 
paradigm has been well characterized in the literature [43]. Notably, binding of miRNA to circRNA molecules does not necessarily constitute miRNA suppression. CircRNA-miRNA complexes may convey susceptibility to degradation, which in turn release miRNA to the intracellular milieu. This was evidenced in a recent report that showed ciRS-7 bound to miR-671 created an Argonaute 2 cleavage site, which released the "passenger" miR-7 in the process and affecting brain function [44]. Therefore, this suggested that circRNAs may function as miRNA reservoirs and facilitate miRNA transportation [45]. Based on our in silico evaluation of miRNA seed sequences present in exons within circRNA structures relative to those exons that do not form part of circRNA, we found no statistical overrepresentation. This suggests that the altered circRNA levels in monocytes, $\mathrm{T}$ cells, and B cells of sepsis patients relative to healthy subjects may partake in cellular functions that are not exclusive to circRNA sponging. Many circRNAs are predicted to harbor RNA binding protein sites [46], with a few reports demonstrating the capacity of circRNA for protein binding. For example, circMBL/MBNL1 generated in both drosophila and humans was demonstrated to specifically and strongly bind to the RNA binding protein muscleblind (MBL/MBNL) [41]. Furthermore, and in line with our findings, circRNA expression was shown to be cell-type-specific [47], which may suggest functionality of circRNA is also dependent on the type of cell and differentiation state. Of note, enucleated blood cells, that is, erythrocytes and platelets, were reported to contain the highest levels of circRNA when compared to all hematopoietic cell lineages [47].

CircRNA detection has been reported to be in part dependent on the detection algorithm. Thus, to ascertain robustness in circRNA detection, it is important to combine bioinformatics and experimental approaches. By leveraging on a fundamental property of circRNA, that is, the lack of a free $3^{\prime}$ end required for digestion with RNase R [13-15], a 3' to 5' exoribonuclease enzyme, it is possible to differentiate linear and circRNA species. In this way, we showed that circRNAs VCAN (chr5: 83519349-83522309) and CHD2 (chr15: 9300051293014909) were detectable in healthy monocytes. While we focused our attention on the two significantly altered circRNAs in CAP compared to health, the presence of other circRNA species in all cell types is certainly not excluded. The limited number of significantly altered circRNA in CAP relative to health can be explained, at least in part, by the low sample size in our discovery phase by RNA-seq. Furthermore, validating circRNA existence and expression in CAP patients would further heighten the robustness of our findings, though the availability of RNA from specific immune cells was limiting. Future studies specifically designed to evaluate
circRNA expression and biology in larger cohorts of patients with sepsis are warranted.

\section{Conclusion}

In conclusion, this is the first study to delineate the expression profiles of circRNAs in specific immune cell types of critically ill patients with sepsis secondary to CAP relative to health. Levels of circRNA were significantly elevated in PBMCs of sepsis patients, with monocytes producing the highest amounts of circRNA relative to CD4+, CD8+ T cells, and B cells. CircRNA expression was significantly altered in patients as compared to healthy subjects, with high specificity for the type of mononuclear cell. By detecting the existence and accumulation of circRNAs in important immune cell subsets, our study adds another layer of regulation to the host response. Therefore, we provide a framework that not only offers new insight into the immunopathology of sepsis but may also represent a novel avenue for drug discovery.

\section{Supplementary information}

Supplementary information accompanies this paper at https://doi.org/10. 1186/s13054-020-03146-4

Additional file 1: Figure S1. Workflow of the analysis of the circular RNAs.

Additional file 2: Figure S2. Schematic of the divergent primers used for the detection of cicrRNAs.

Additional file 3: Table S1. Table of differentially abundant circRNAs.

Additional file 4: Figure S3. Linear RNA transcriptional changes in monocytes, CD4+ and CD8+ T-cells and CD19+ B-cells of CAP patients compared to healthy subjects.

\section{Abbreviations}

circRNA: Circular RNA; PBMCs: Peripheral blood mononuclear cells; CAP: Community-acquired pneumonia; RCS: Reverse complementary sequence; miRNA: MicroRNA; RIN: RNA integrity number; PCA: Principal component analysis

\section{Acknowledgements}

The authors thank all patients and volunteers who participated in this study as well as the critical care nursing staff at both the Academic Medical Centre and University Medical Centre Utrecht ICUs.

\section{Authors' contributions}

B.P.S. conceived and supervised the study. B.P.S., H.N.K., S.A., and A.H.Z. analyzed and interpreted the RNA-seq data. A.J.H. and B.P.S. purified cells and isolated RNA. X.B. and A.M.K. performed the PCR validation experiments. X.B., T.S.R.E., B.W.H., L.A.V., J.H., and M.J.S. included patients and healthy volunteers and recorded clinical data. T.vd. P. secured the funding for this study. B.P.S. and H.N.K. wrote the manuscript with feedback from all other authors. The authors read and approved the final manuscript.

\section{Funding}

This study was funded by CTMM, the Center for Translational Molecular Medicine (www.ctmm.nl; grant 04l-201).

Availability of data and materials

Data are accessible in the National Center for Biotechnology Information Gene Expression Omnibus [48] accession number GSE136200. 


\section{Ethics approval and consent to participate}

This study was part of the Molecular Diagnosis and Risk Stratification of sepsis (MARS) project, a prospective observational study in the mixed ICUs of two tertiary teaching hospitals in the Netherlands (Academic Medical Center, Amsterdam, and University Medical Center Utrecht, Utrecht) (ClinicalTrials. gov identifier NCT01905033). The institutional review boards of both participating centers approved an opt-out consent method (IRB No. 10056C). Written informed consent was provided by all healthy volunteers. All procedures complied with the Helsinki Declaration of 1975 (revised 1983).

\section{Consent for publication}

All authors reviewed and provided consent to publish the work described in this study.

\section{Competing interests}

All authors declare no conflicts of interest.

\section{Author details}

${ }^{1}$ Center for Experimental Molecular Medicine, Amsterdam University Medical Centers, location Academic Medical Center, University of Amsterdam, Room G2-105, Meibergdreef 9, 1105 AZ Amsterdam, the Netherlands. ${ }^{2}$ Department of Clinical Epidemiology, Biostatistics and Bioinformatics, Amsterdam University Medical Centers, location Academic Medical Center, University of Amsterdam, Amsterdam, the Netherlands. ${ }^{3}$ Department of Intensive Care Medicine, Amsterdam University Medical Centers, location Academic Medical Center, University of Amsterdam, Amsterdam, the Netherlands. ${ }^{4}$ Mahidol Oxford Tropical Medicine Research Unit, Bangkok, Thailand. ${ }^{5}$ Division of Infectious Diseases, Amsterdam University Medical Centers, location Academic Medical Center, University of Amsterdam, Amsterdam, the Netherlands.

Received: 15 January 2020 Accepted: 3 July 2020

Published online: 13 July 2020

\section{References}

1. Angus DC, van der Poll T. Severe sepsis and septic shock. N Engl J Med. 2013;369(9):840-51.

2. Singer M, Deutschman CS, Seymour CW, Shankar-Hari M, Annane D, Bauer M, Bellomo R, Bernard GR, Chiche JD, Coopersmith CM, et al. The third international consensus definitions for sepsis and septic shock (Sepsis-3). JAMA. 2016;315(8):801-10.

3. Marshall JC. Why have clinical trials in sepsis failed? Trends Mol Med. 2014; 20(4):195-203.

4. van der Poll T, van de Veerdonk FL, Scicluna BP, Netea MG. The immunopathology of sepsis and potential therapeutic targets. Nat Rev Immunol. 2017;17(7):407-20.

5. Hotchkiss RS, Monneret G, Payen D. Sepsis-induced immunosuppression: from cellular dysfunctions to immunotherapy. Nat Rev Immunol. 2013; 13(12):862-74

6. Davenport EE, Burnham KL, Radhakrishnan J, Humburg P, Hutton P, Mills TC, Rautanen A, Gordon AC, Garrard C, Hill AV, et al. Genomic landscape of the individual host response and outcomes in sepsis: a prospective cohort study. Lancet Respir Med. 2016;4(4):259-71.

7. Cheng SC, Scicluna BP, Arts RJ, Gresnigt MS, Lachmandas E, GiamarellosBourboulis EJ, Kox M, Manjeri GR, Wagenaars JA, Cremer OL, et al. Broad defects in the energy metabolism of leukocytes underlie immunoparalysis in sepsis. Nat Immunol. 2016;17(4):406-13.

8. Scicluna BP, van Vught LA, Zwinderman AH, Wiewel MA, Davenport EE, Burnham KL, Nurnberg P, Schultz MJ, Horn J, Cremer OL, et al. Classification of patients with sepsis according to blood genomic endotype: a prospective cohort study. Lancet Resp Med. 2017;5(10):816-26.

9. Chen LL. The biogenesis and emerging roles of circular RNAs. Nat Rev Mol Cell Biol. 2016;17(4):205-11.

10. Cocquerelle C, Mascrez B, Hetuin D, Bailleul B. Mis-splicing yields circular RNA molecules. FASEB J. 1993;7(1):155-60.

11. Salzman J, Chen RE, Olsen MN, Wang PL, Brown PO. Cell-type specific features of circular RNA expression. PLoS Genet. 2013:9(9):e1003777.

12. Salzman J, Gawad C, Wang PL, Lacayo N, Brown PO. Circular RNAs are the predominant transcript isoform from hundreds of human genes in diverse cell types. PLoS One. 2012;7(2):e30733.
13. Guo JU, Agarwal V, Guo H, Bartel DP. Expanded identification and characterization of mammalian circular RNAs. Genome Biol. 2014;15(7):409.

14. Zhang Y, Xue W, Li X, Zhang J, Chen S, Zhang JL, Yang L, Chen LL. The biogenesis of nascent circular RNAs. Cell Rep. 2016;15(3):611-24.

15. Li $X$, Yang $L$, Chen LL. The biogenesis, functions, and challenges of circular RNAs. Mol Cell. 2018;71(3):428-42.

16. Klein Klouwenberg PM, Ong DS, Bos LD, de Beer FM, van Hooijdonk RT, Huson MA, Straat M, van Vught LA, Wieske L, Horn J, et al. Interobserver agreement of Centers for Disease Control and Prevention criteria for classifying infections in critically ill patients. Crit Care Med. 2013;41(10):2373-8.

17. Scicluna BP, Klein Klouwenberg PM, van Vught LA, Wiewel MA, Ong DS, Zwinderman AH, Franitza M, Toliat MR, Nurnberg P, Hoogendijk AJ, et al. A molecular biomarker to diagnose community-acquired pneumonia on intensive care unit admission. Am J Respir Crit Care Med. 2015;192(7):826-35.

18. Calandra T, Cohen J. The international sepsis forum consensus conference on definitions of infection in the intensive care unit. Crit Care Med. 2005; 33(7):1538-48.

19. Zimmerman JE, Kramer AA, McNair DS, Malila FM. Acute Physiology and Chronic Health Evaluation (APACHE) IV: hospital mortality assessment for today's critically ill patients. Crit Care Med. 2006;34(5):1297-310.

20. Vincent JL, Moreno R, Takala J, Willatts S, DeMendonca A, Bruining H, Reinhart CK, Suter PM, Thijs LG. The SOFA (sepsis-related organ failure assessment) score to describe organ dysfunction/failure. Intensive Care Med. 1996;22(7):707-10

21. Bolger AM, Lohse M, Usadel B. Trimmomatic: a flexible trimmer for Illumina sequence data. Bioinformatics. 2014;30(15):2114-20.

22. Harrow J, Denoeud F, Frankish A, Reymond A, Chen CK, Chrast J, Lagarde J, Gilbert JG, Storey R, Swarbreck D, et al. GENCODE: producing a reference annotation for ENCODE. Genome Biol. 2006;7(Suppl 1):S4 1-9.

23. Kim D, Pertea G, Trapnell C, Pimentel H, Kelley R, Salzberg SL. TopHat2: accurate alignment of transcriptomes in the presence of insertions, deletions and gene fusions. Genome Biol. 2013;14(4):R36.

24. Liao Y, Smyth GK, Shi W. featureCounts: an efficient general purpose program for assigning sequence reads to genomic features. Bioinformatics. 2014;30(7):923-30

25. Love Ml, Huber W, Anders S. Moderated estimation of fold change and dispersion for RNA-seq data with DESeq2. Genome Biol. 2014;15(12):550.

26. Wang $K$, Singh D, Zeng Z, Coleman SJ, Huang Y, Savich GL, He X, Mieczkowski P, Grimm SA, Perou CM, et al. MapSplice: accurate mapping of RNA-seq reads for splice junction discovery. Nucleic Acids Res. 2010;38(18): e178.

27. Langmead B, Trapnell C, Pop M, Salzberg SL. Ultrafast and memory-efficient alignment of short DNA sequences to the human genome. Genome Biol. 2009:10(3):R25.

28. Morgan M, Anders S, Lawrence M, Aboyoun P, Pages H, Gentleman R. ShortRead: a bioconductor package for input, quality assessment and exploration of high-throughput sequence data. Bioinformatics. 2009;25(19): 2607-8

29. Aufiero S, van den Hoogenhof MMG, Reckman YJ, Beqqali A, van der Made I, Kluin J, Khan MAF, Pinto YM, Creemers EE. Cardiac circRNAs arise mainly from constitutive exons rather than alternatively spliced exons. Rna. 2018; 24(6):815-27.

30. Smit AFA HRG, P: RepeatMasker Open-4.0. http://www.repeatmasker.org/ (Accessed 2 May 2017).

31. Kruger J, Rehmsmeier M. RNAhybrid: microRNA target prediction easy, fast and flexible. Nucleic Acids Res. 2006;34:W451-4.

32. Tan WL, Lim BT, Anene-Nzelu CG, Ackers-Johnson M, Dashi A, See K, Tiang Z, Lee DP, Chua WW, Luu TD, et al. A landscape of circular RNA expression in the human heart. Cardiovasc Res. 2017;113(3):298-309.

33. Karolchik D, Hinrichs AS, Furey TS, Roskin KM, Sugnet CW, Haussler D, Kent WJ. The UCSC Table Browser data retrieval tool. Nucleic Acids Res. 2004; 32(Database issue):D493-6.

34. Ashburner M, Ball CA, Blake JA, Botstein D, Butler H, Cherry JM, Davis AP, Dolinski K, Dwight SS, Eppig JT, et al. Gene ontology: tool for the unification of biology. The Gene Ontology Consortium. Nat Genet. 2000;25(1):25-9.

35. Vincent HA, Deutscher MP. Substrate recognition and catalysis by the exoribonuclease RNase R. J Biol Chem. 2006;281(40):29769-75.

36. Ruijter JM, Pfaffl MW, Zhao S, Spiess AN, Boggy G, Blom J, Rutledge RG, Sist D, Lievens A, De Preter K, et al. Evaluation of qPCR curve analysis methods for reliable biomarker discovery: bias, resolution, precision, and implications. Methods. 2013;59(1):32-46. 
37. Memczak S, Jens M, Elefsinioti A, Torti F, Krueger J, Rybak A, Maier L, Mackowiak SD, Gregersen LH, Munschauer M, et al. Circular RNAs are a large class of animal RNAs with regulatory potency. Nature. 2013;495(7441):333-8.

38. Hansen $\mathrm{TB}$, Jensen $\mathrm{Tl}$, Clausen BH, Bramsen JB, Finsen B, Damgaard CK, Kjems J. Natural RNA circles function as efficient microRNA sponges. Nature. 2013:495(7441):384-8.

39. Yang $Y$, Fan $X$, Mao M, Song X, Wu P, Zhang Y, Jin Y, Yang Y, Chen LL, Wang $Y$, et al. Extensive translation of circular RNAs driven by N (6)methyladenosine. Cell Res. 2017;27(5):626-41.

40. Yang Y, Gao X, Zhang M, Yan S, Sun C, Xiao F, Huang N, Yang X, Zhao K, Zhou $\mathrm{H}$, et al. Novel role of FBXW7 circular RNA in repressing glioma tumorigenesis. J Natl Cancer Inst. 2018;110(3):304-15.

41. Ashwal-Fluss R, Meyer M, Pamudurti NR, Ivanov A, Bartok O, Hanan M, Evantal N, Memczak S, Rajewsky N, Kadener S. circRNA biogenesis competes with pre-mRNA splicing. Mol Cell. 2014;56(1):55-66

42. Liang D, Tatomer DC, Luo Z, Wu H, Yang L, Chen LL, Cherry S, Wilusz JE. The output of protein-coding genes shifts to circular RNAs when the premRNA processing machinery is limiting. Mol Cell. 2017;68(5):940-54 e943.

43. Haddad G, Lorenzen JM. Biogenesis and function of circular RNAs in health and in disease. Front Pharmacol. 2019;10:428.

44. Piwecka M, Glazar P, Hernandez-Miranda LR, Memczak S, Wolf SA, RybakWolf A, Filipchyk A, Klironomos F, Cerda Jara CA, Fenske P, et al. Loss of a mammalian circular RNA locus causes miRNA deregulation and affects brain function. Science. 2017;357(6357):eaam8526.

45. Kristensen LS, Hansen TB, Veno MT, Kjems J. Circular RNAs in cancer: opportunities and challenges in the field. Oncogene. 2018;37(5):555-65.

46. Du WW, Zhang C, Yang W, Yong T, Awan FM, Yang BB. Identifying and characterizing circRNA-protein interaction. Theranostics. 2017;7(17):4183-91.

47. Nicolet BP, Engels S, Aglialoro F, van den Akker E, von Lindern M, Wolkers MC. Circular RNA expression in human hematopoietic cells is widespread and cell-type specific. Nucleic Acids Res. 2018;46(16):8168-80.

48. Edgar R, Domrachev M, Lash AE. Gene Expression Omnibus: NCBI gene expression and hybridization array data repository. Nucleic Acids Res. 2002; 30(1):207-10.

\section{Publisher's Note}

Springer Nature remains neutral with regard to jurisdictional claims in published maps and institutional affiliations.

Ready to submit your research? Choose BMC and benefit from:

- fast, convenient online submission

- thorough peer review by experienced researchers in your field

- rapid publication on acceptance

- support for research data, including large and complex data types

- gold Open Access which fosters wider collaboration and increased citations

- maximum visibility for your research: over $100 \mathrm{M}$ website views per year

At $\mathrm{BMC}$, research is always in progress.

Learn more biomedcentral.com/submissions 